\title{
Los derechos de las personas con discapacidad en la Corte Suprema: Sandoval con Empresa de Transporte de Pasajeros Metro S.A.
}

The rights of persons with disabilities in The Supreme Court: Sandoval with Transportation Company of Pasajeros metro s.a.

\author{
Benjamín GAJARDO ${ }^{1}$ \\ Universidad Andrés Bello \\ benjamin.gajardo@unab.cl
}

\begin{abstract}
Resumen: El siguiente comentario de jurisprudencia analiza una sentencia de la Corte Suprema que discute la discriminación de una persona con discapacidad. En la presente sentencia, la Corte Suprema analiza la calificación de discriminación en las instancias anteriores, en donde rechaza la interpretación de discriminación utilizada y, en conjunto a ello, ofrece un nuevo marco conceptual de discriminación en las personas con discapacidad.
\end{abstract}

Palabras clave: Corte Suprema, discapacidad, discriminación.

1 Abogado. Profesor instructor. Universidad Andrés Bello. Facultad de Derecho, Bellavista 0121, Providencia 7500000. Correspondencia a: benjamin.gajardo@unab.cl. Agradezco la valiosa asistencia y comentarios de mi ayudante de investigación Felipe Núñez. 


\begin{abstract}
The present jurisprudence comment analyzes a ruling of the Supreme Court that discusses discrimination against a person with disabilities. In this judgment, the Supreme Court analyzes the classification of discrimination in the previous instances, where it rejects the interpretation of discrimination used and, in conjunction with it, offers a new conceptual framework of discrimination in persons with disabilities.
\end{abstract}

Keywords: Supreme Court, disability, discrimination.

\title{
1. Introducción
}

En el siguiente artículo evalúo el rendimiento argumentativo de la sentencia pronunciada por la Excelentísima Corte Suprema con fecha 25 de julio 2018, el fallo causa rol 20.679.2016 (en adelante el fallo o la sentencia), referida a un recurso de casación en el fondo sobre una acción de no discriminación de la Ley 20.609. La presente, revierte la decisión de primera y segunda instancia fallando, en consecuencia, a favor de la persona discriminada.

La relevancia del fallo y, por tanto, la razón de su selección, se asienta en dos motivos principalmente: el primero, consiste en que esta sentencia es uno de los escasos casos que han llegado a la Corte Suprema en lo relativo a la acción de no discriminación arbitraria²; segundo, la decisión de la corte marca lineamientos y directrices importantes en lo relativo al derecho antidiscriminatorio aplicado en las personas con discapacidad (en adelante $\mathrm{PcD}$ ) en nuestro sistema jurídico, en donde será posible visualizar principios y criterios para resolución de conflictos.

El propósito de este análisis se centrará en los aspectos sustantivos del fallo como también en los aspectos controvertidos de las instancias precedentes a la decisión de la Corte Suprema. De tal modo, este trabajo contendrá la siguiente estructura: en primer lugar, se analizarán los hechos del caso; luego, se abordarán los argumentos centrales de la Corte Suprema y, finalmente, se ofrecerá un comentario crítico.

2 Díaz de Valdés (2018). 


\section{El caso}

\subsection{Los hechos}

De acuerdo con la demanda, los hechos del caso ocurrieron el 19 de mayo 2016 aproximadamente a las 07.15 a.m. de la mañana, en circunstancias que Jeanette Sandoval, demandante del caso y persona con discapacidad física de un $70 \%$, se encontraba realizando su viaje diario en el Metro de Santiago. En dicho contexto, el carro del metro frena bruscamente y comunica a sus pasajeros que se iniciará un operativo de seguridad por aviso de un posible artefacto explosivo en la estación. Como consecuencia de lo anterior, comienza la evacuación de las personas abordo; por consiguiente, Jeanette procedió a evacuar, pero dicha acción se vio truncada debido a que el salvaescala, artefacto de accesibilidad para las PcD instalado en el metro, estaba en mal estado. Lo relatado, provocó que Jeannette, naturalmente, no pudiera evacuar junto al resto de pasajeros y tuvo que permanecer dentro de la estación de metro durante el operativo en cuestión.

Estos hechos motivaron la interposición de una acción antidiscriminación de la Ley 20.609 por parte de Jeanette Sandoval en contra de Metro, acusando una discriminación arbitraria por las acciones del personal de la empresa.

\subsection{Sentencia en primera y segunda instancia}

La sentencia, en primera instancia, emanó del $29^{\circ}$ Juzgado Civil de Santiago, tribunal que revisó la acción antidiscriminación de Jeannette Sandoval en contra Metro. La base de la demanda se funda en que la discriminación se establece debido a que los mecanismos de accesibilidad estaban defectuosos; así pues, no podían ser utilizados por Jeannette generando una diferencia arbitraria respecto al resto de los pasajeros. De esta manera, la demandante arguye que, "(...) se sintió discriminada y humillada por parte de la demanda, en atención a que por el solo hecho de estar en silla de ruedas, se le impuso la carga de exponerse a un hecho que pudo tener consecuencias trágicas, y asimismo a la carga de tener que llegar tarde a sus actividades. Lógicamente, tal carga no se 
verificó respecto de las personas que no tiene discapacidad". Por ende, y de acuerdo con el razonamiento de la demandante, estos hechos deben ser calificados como discriminatorios en relación con el artículo $2^{\circ}$ de la ley 20.609 .

Por su parte, la parte demandada, no discute en profundidad si las circunstancias descriptas son o no discriminatorios, sino que, se defiende argumentando que Metro no se encuentra en la obligación legal de otorgar accesibilidad, debido al plazo conferido en el inciso cuarto del articulo primero transitorio de la Ley 20.422. En consecuencia, Metro afirma “(...) el acceso a los medios de transporte público de pasajeros (...) deberá ser accesible y utilizables en forma autovalente y sin dificultad por personas con discapacidad, especialmente aquellas con movilidad reducida, dentro del plazo de años ocho contado desde la publicación de esta Ley en el Diario Oficial"; de esta forma, la obligación legal de accesibilidad aún no es exigible para Metro.

Ante esta exposición de argumentos la Corte de primera instancia afirma que no es posible acreditar un trato carente de racionalidad contra Jeannette, pues i) no se acredito fehacientemente, por parte de la demandada, que la salvaescala se encontraba defectuoso como tampoco un trato injustificado; ii) a pesar de lo dicho en el punto anterior, de acuerdo con la Ley 20.422 no existe obligación legal para Metro de contar con mecanismos de accesible para las PcD. Por otra parte, esta misma argumentación fue apoyada por la Corte de Apelaciones ratificando, así, la decisión de primera instancia.

\section{Argumentos de la Corte Suprema}

A partir de un recurso de casación en el fondo por la parte demandante, la Corte Suprema conoce el asunto, y revierte lo fallado en las instancias anteriores, resolviendo a favor de la PcD. De esta manera, los argumentos utilizados por la Corte Suprema son:

a) El deber de accesibilidad: Metro al iniciar un extenso y robusto programa de accesibilidad en sus instalaciones para las $P c D$, es posible colegir que ya renunció tácitamente al plazo legal de la Ley 20.422. De tal 
modo, se rechaza lo argumentado por Metro en las instancias anteriores y se torna exigible la obligación de accesibilidad. En este orden de ideas, sostener que no existe la obligación legal de accesibilidad una vez ya comenzado un programa de acceso implica contravenir el "deber de no regresividad de los derechos humanos".

b) Discriminación: Se sostiene que un error en la interpretación respecto a la calificación de discriminación del artículo 2 de la Ley 20.609, puesto que "(...) un plan de evacuación implica sacar a todos los pasajeros sin distinción, ya que corre riesgo el derecho a la vida o integridad física. Entonces, dejar a una persona con discapacidad esperando en el andén de una estación, en una situación de emergencia, dado el estado del salvaescala, importa una acción de distinción y exclusión". Por consiguiente, la Corte sostiene que la denegación de ajustes razonable importa una discriminación, recogiendo, así, lo estipulado en el artículo 2 de la Convención sobre Derechos de las Personas con Discapacidad.

Por lo tanto, la Corte Suprema acoge el recurso de casación en el fondo por la parte demandante y concluye, en virtud de los argumentos descritos, que si existió un acto discriminatorio por parte de Metro en contra de Jeannette Sandoval.

\section{Comentario}

El objeto de este comentario es la configuración normativa de la discriminación en las PcD. En efecto, la presente sentencia nos ofrece criterios y estándares que permiten atisbar como opera la discriminación en las personas con discapacidad. Posteriormente, y una vez ordenadas las piezas del puzzle, pasaré a realizar una evaluación crítica de la decisión de la Corte Suprema.

En primer lugar, es posible sostener que la Corte Suprema comprende que la accesibilidad es un criterio relevante al momento de analizar una correcta interpretación de la discriminación en las personas con discapacidad. En efecto, la relación discriminación y accesibilidad responde, como sostiene Jerzy Grzeda, a que "(...) las personas con discapacidad enfrentan un conjunto de problemas que ningún otro grupo [discrimina- 
do] encuentra: las barreras en el entorno"3; por ello, las acciones de corte antidiscriminatorios en las personas con discapacidad demandan la supresión de estas barreras de entorno para que las personas puedan desarrollar una plena participación en sociedad, así "La discapacidad se puede eliminar al reducir estas barreras"4. Por otra parte, el Comité sobre los Derechos de las Personas con Discapacidad de la Organización de las Naciones Unidas ONU ha sostenido que, "La accesibilidad es una condición previa para que las personas con discapacidad puedan vivir en forma independiente y participar plenamente en la sociedad en igualdad de condiciones"; más aún, ha afirmado que:

"Este enfoque se basa en la prohibición de la discriminación; debe considerarse que la denegación de acceso constituye un acto discriminatorio, independientemente de que quien lo cometa sea una entidad pública o privada. Deben tener accesibilidad todas las personas con discapacidad, con independencia del tipo de discapacidad y de su situación jurídica o social, sexo o edad" 5 .

Dicha compresión denota asimilar el cambio de paradigma de la discapacidad, consistente en el paso del "modelo médico o rehabilitador" al modelo de "social o de derechos" 6 . Este giro de enfoque implica una ruptura en el tratamiento de la discapacidad, pues, en el modelo médico, las discapacidades se les asociaba a un problema médico; en cambio, en el modelo de derechos humanos el foco son las barreras existentes en la sociedad que impiden y dificultan la participación de estas en la sociedad $^{7}$, este modelo se marca con la aprobación de la Convención de Derechos de las Personas con Discapacidad. En tal sentido, es posible establecer, en que lo respecta a las personas con discapacidad, una relación entre accesibilidad y la no discriminación, como razona Cayo, "La ausencia de accesibilidad, que frustra de ordinario el ejercicio regular de los derechos a las personas con discapacidad, se reputa en este sistema

3 GRZEDA (1994), pp. 145-147.

4 ÍDEM (1994).

5 CRPD/C/11/3 (2013), p. 4. El énfasis es propio.

6 Oliver (1996), pp. 30-42.

7 Quinn y Degener (2002), p. 20. 
como discriminación que atenta contra la igualdad"8. En esta misma línea argumentativa, Broderick ha concluido que el alcance de la accesibilidad va más allá que una mera medida u orientación para los estados, sino pues, la obligación de accesibilidad derivada de la Convención demanda a la realización de medidas concretas, ello, debido a que dicha obligación se vincula al derecho a la igualdad y no discriminación ${ }^{9}$.

Por otra parte, y como segundo comentario, es posible afirma que la Corte Suprema incorpora tibiamente dentro de la articulación de discriminación en las personas con discapacidad la noción de ajustes razonables. Esta idea proviene, tal como indica la corte, del artículo $2^{\circ}$ de la Convención de Derechos de las Personas con Discapacidad, el que señala una forma de discriminación consiste en la "(...) denegación de ajustes razonables", posteriormente la Convención define el concepto de ajustes razonables como:

"Por "ajustes razonables" se entenderán las modificaciones y adaptaciones necesarias y adecuadas que no impongan una carga desproporcionada o indebida, cuando se requieran en un caso particular, para garantizar a las personas con discapacidad el goce o ejercicio, en igualdad de condiciones con las demás, de todos los derechos humanos y libertades fundamentales".

Por ende, la idea de ajustes razonables se nos devela como una construcción conceptual de medida de acción afirmativa de baja intensidad que permite evitar discriminaciones hacia las PcD. Sobre lo dicho, Finsterbusch describe los ajustes razonables como:

“(... la construcción jurídica que se encuentra al servicio de la igualdad. En efecto, los ajustes razonables hacen un llamado a la imaginación conceptual, en donde la idea de tratamiento diferenciado se vuelve necesaria en pro de exigencias de una igualdad material o efectiva. En este sentido, corresponde a una construcción intelectual que busca evitar la discriminación indirecta"10.

8 CAYO (2012), p. 3.

9 BRODERICK (2019), p. 4.

10 FINSTERBUSCH (2016), p. 228. 
A través de esta herramienta jurídica es posible evitar futuras discriminaciones arbitrarias sin desplegar una carga desproporcionada; así, los ajustes razonables constituyen una relevante herramienta en el derecho antidiscriminatorio. En esta línea, Díaz de Valdés afirma que:

“(...) la acomodación razonable intenta incluir o incorporar a personas que presentan alguna diferencia que, de no mediar la acomodación quedarían excluidas de ciertas situaciones o del ejercicio de ciertos derechos. Por los mismo, el énfasis se encuentra en alcanzar soluciones prácticas, concretas, que logren ese objetivo, lo que su vez pone el acento en la igualdad material que se desea lograr más que la igualdad formal del trato"11.

Entonces, tanto la idea de accesibilidad como los ajustes razonables son piezas importantes para la determinación de la discriminación en las personas con discapacidad. Al respecto, cabe realizar la siguiente reflexión, ¿qué conexión o relación existe entre la accesibilidad y los ajustes razonables? Sobre lo consultado, es posible comprender que ambas ideas responden a un haz de deberes jurídicos que buscan el desarrollo de bienestar de las $\mathrm{PcD}$, es decir, una primera aproximación es que la no discriminación en las $\mathrm{PCD}$, particularmente, busca que los participantes de dicho grupo consigan grados de bienestar relevantes o satisfactorios.

En otro orden de ideas, y bajo el supuesto que la accesibilidad y los ajustes razonables derivan del mandato de no discriminación, es posible, por tanto, sostener que ambas ideas responden al mismo objetivo; así, la accesibilidad universal representa el fin último buscado, de tal forma, tanto la accesibilidad como los ajustes razonables son forma de conseguir, con distinta intensidad, este ideario o ideal regulativo.

Así las cosas, la accesibilidad universal demanda la eliminación total de barreras que dificulten o impidan el acceso de las $\mathrm{PcD}$ en algún ámbito de la vida. Más aún, la Convención de Derechos de las Personas con Discapacidad comprende que la accesibilidad se compone por tres derechos: i) el derecho a una vida independiente; ii) a una participación plena

11 Díaz de Valdés (2019), p. 215. 
en la sociedad y iii) la igualdad de oportunidades. Por consiguiente, la accesibilidad universal constituye el mandato de optimización ${ }^{12}$ el cual debe ser materializado en forma de obligaciones positivas. En consecuencia, la accesibilidad responde la mejor forma de optimizar dicho mandato, pues las acciones de accesibilidad importan relevantes modificaciones para la eliminación de las barreras que impiden el goce de los derechos, en cambio, los ajustes razonables operan cuando “(...) el dispositivo genérico de la accesibilidad universal y el diseño para todas las personas cesa, no alcanza a la situación particular que experimenta la PcD. Ante el fracaso garantistas, valga la expresión, de la accesibilidad universal y del diseño para todas las personas, que ha de gozar de precedencia, se despliegan los ajustes razonables ${ }^{13}$ ". En otras palabras, la accesibilidad se nos muestra como un derecho de primer orden, mientras que, y a falta del primer, los ajustes razonables desempeñan derechos de segundo orden; una idea similar sostiene Cayo, "(...) en el esquema general de la igualdad de derechos de las personas con discapacidad, los ajustes razonables son subsidiarios, operan en defecto de la accesibilidad universal y sus correlatos, que representarían la garantía reforzada o de primer grado" 14 .

Por otro lado, la Corte Suprema rechaza la tesis adherida en las instancias anteriores, relativa a que Metro no se encontraba en la obligación legal de dar acceso. Sobre lo dicho, la Corte Suprema sostiene que Metro abandonó el plazo legal para el cumplimiento del artículo 28 de la Ley $\mathrm{N}^{\circ} 19.284^{15}$, dado que Metro ya comenzó con el acceso gradual en sus instalaciones, por tanto, hizo una renuncia tacita al plazo legal mencionado. Esto nos ofrece una compresión de la corte sobre la obligación legal de acceso a las $\mathrm{PcD}$, más aún, cuando el argumento más común en la litigación de la discriminación por discapacidad es la eludir la obligación legal de accesibilidad.

12 Alexy (1993).

13 CAYo (2012), p. 5. El énfasis es propio.

14 ÍDEM (2012), p. 5.

15 El plazo legal referido por la corte se encuentra el Decreto Supremo $N^{\circ} 50$. 


\section{1. ¿Fue correcta la decisión de la Corte?}

En lo que respecta a la decisión de la Corte Suprema antes es necesario, naturalmente, referirse brevemente a las instancias anteriores. Desde un punto de vista descriptivo, es posible señalar que tanto el $29^{\circ}$ Juzgado Civil de Santiago como la Corte de Apelaciones de Santiago utilizan la misma base discursiva para analizar la acción de no discriminación. La posición de estas cortes consiste en rechazar la acción antidiscriminación, es decir, calificar la no existencia de actos discriminatorios por parte de Metro, debido a que no existieron, en su razonamiento, "(...) actos carentes de racionalidad en términos arbitrarios". Como apoyo a la articulación argumentativa, se suma la tesis que Metro no se encontraba, aún, en plazo legal que permitiera hacer exigible la obligación de accesibilidad en sus instalaciones. En resumen: i) una endeble interpretación de discriminación; ii) una mirada formalista sobre la obligación legal de acceso para las PcD.

Desde el enfoque de los derechos humanos de las personas con discapacidad $^{16}$ y a partir de articulado en el apartado anterior, lo planteado por las cortes es insuficiente, pues califican la discriminación desde una perspectiva negativa, a saber, al no encontrar una acción o restricción -no razonable- en contra de las personas con discapacidad, entonces, no existe la discriminación, pero, el juicio normativo de no discriminación en las personas con discapacidad demanda articulaciones diferentes.

Como se observó anteriormente, la discriminación sobre el grupo estudiado implica incorporar una dimensión positiva en el análisis. Por lo tanto, la negación de dicha dimensión positiva conduce a un resultado jurídicamente incorrecto a la luz de la teoría de los derechos de las personas con discapacidad ${ }^{17}$, especialmente, desde la compresión de la modelo social de la discapacidad. Esta interpretación más robusta de discriminación cobra sentido, especialmente, en las PcD, cuando los estudios y datos han señalado que este grupo social suele sufrir, generalmente, discri-

16 De Asis comenta que asumir la discapacidad como un asunto de derechos humanos implica asumir que el sistema jurídico es responsable, en cierta medida, de las barreras paras las PcD.

17 De AsIs (2013). 
minaciones indirectas o neutrales ${ }^{18}$. En atención a lo esgrimido, Díaz de Valdés constante una fuerte reticencia, por las cortes y políticas públicas, en asumir un compromiso por eliminar este tipo de discriminaciones. ${ }^{19}$ El ejemplo más significativo es que nuestra Ley Antidiscriminación omite este elemento, dejando a la discrecionalidad del juez su aplicación.

Por su parte, la Corte Suprema suma en su razonamiento la Convención sobre los Derechos de las Personas con Discapacidad, esto, añade en su análisis conceptos no previstos en las instancias anteriores. Lo subscrito, transforma el análisis de la idea de no discriminación en una compresión más robusta, como bien aprecia Agustina Palacios "(...) las consecuencias jurídicas de esta sustitución del modelo rehabilitador por el modelo social se encuentran estrechamente ligadas al derecho a la igualdad, que comprende dentro de su contenido ciertas nociones esenciales, como son la prohibición de discriminación indirecta y la idea de ajustes razonables"20. Así pues, lo descrito explica por qué la Corte Suprema realiza otra forma de determinación normativa de la discriminación y, por ende, llega a otro resultado, más favorable para la PcD. En efecto, la compresión de la "razonabilidad" cambia drásticamente al integrar en el razonamiento la Convención sobre los Derechos de las Personas con Discapacidad; en palabra de la corte:

"Tratándose del segundo, que la acción carezca de fundamento razonable, no puede ser calificado sensato el argumento invocado por el demandado para justificar la acción de distinción, en este caso, dejar a la demandante como se expresó, más aún si, como se evidenció, había comenzado con el plan de accesibilidad. En tal sentido, la falta de "ajustes razonables" importan una discriminación, así lo establece el artículo 2 de la Convención sobre Derechos de las Personas con Discapacidad".

En definitiva, la Corte Suprema incorpora elementos del modelo social de discapacidad, acogiendo el recurso de casación y revertiendo la decisión.

18 Doyle (2007). Asimismo, el Comité Comité sobre los Derechos de las Personas con Discapacidad, en su Observación general núm. 3, enfatiza la necesidad de preocuparse por la discriminación indirecta o neutrales. CRPD/C/GC/3. (2016), p. 6.

19 Díaz de Valdés (2019), p. 275-276.

20 Palacios (2004), p. 187. 


\section{Conclusiones}

La evaluación de rendimiento de la Corte Suprema es posible considerarla como positiva, sin perjuicio de ello, este hecho no demuestra avances cuantitativos para este grupo social, sino más bien, el mero cumplimiento de obligaciones remotas. Pero, y más importante aún, se constata las grandes dificultades que tiene las PcD en la litigación antidiscriminatoria, así, esta decisión marca la tendencia de resultado de la discriminación en las PcD bajo la Ley 20.60921. En relación a lo anterior, exploraré tres razones que intentan explicar las dificultades de las PcD en el reconocimiento de sus derechos en la litigación: i) las deficiencias propias de la Ley 20.609, particularmente, el concepto legal de acción de no discriminación y las implicancias de las categorías sospechosas; ii) la dispersión normativa de la institucionalidad protectora de las PCD en nuestro sistema jurídico; iii) el formalismo en la adjudicación en las cortes de primera instancia, sumado a la resistencia de aplicar tratados internacional de derechos humanos.

En relación con el primer asunto comentado, la literatura nacional ha cultivado un conjunto relevante de críticas desde hace años a la Ley $20.609^{22}$, pero, y para efecto de esta investigación, me interesa concentrarme en las deficiencias conceptuales en el diseño de la "discriminación" y variables que tengan incidencia en las PcD. De tal modo, la literatura advierte que la definición de discriminación arbitraria no es idónea como categoría normativa, dado que la definición legal sobrecarga conceptos y acepciones de modo innecesario y poco eficiente para un proceso adjudicativo ${ }^{23}$; por otro lado, no existe claridad sobre el rol y la función de las "categorías sospechosas" en la Ley 20.609, como señala Díaz de Valdés "Nos encontramos frente a una redacción altamente deficiente que genera diversas confusiones y que evidencia poca claridad

21 Muñoz (2015); Díaz de Valdés (2017).

22 Alvear y Covarrubias (2012); Vial (2013); Rosales (2014), pp. 255-296; Díaz (2013), pp. 635668; Díaz de Valdés (2013); MuÑoz (2015); EsPARZA (2019).

23 DíAZ de VALdÉs (2013). 
respecto de la intención del legislador"24, ello hace perder la potencia que pueden tener estas categorías en cuanto a medidas deferente para grupos vulnerables. Si bien estas reflexiones críticas tienen, como es posible notar, incidencias empíricas, ellas las que son posibles comprobar en razón del rendimiento de las sentencia de primera instancia de la Ley antidiscriminación.

Como segunda razón, la dispersión normativa de los derechos de los $\mathrm{PcD}$ en nuestro sistema jurídico es un aspecto problemático para una efectiva tutela de los derechos de las PcD. La introducción de normativa deferente hacía las $\mathrm{PcD}$ ha sido de forma lenta, sin orden, con escasa difusión y déficit de mecanismos necesarios para su efectiva realización (enforcement), asimismo, la misma se encuentra distribuida por diferentes campos jurídicos con pocos niveles de consistencia. Todo lo anterior provoca una gran falta de certeza jurídica. Ahora bien, si bien es cierto los sistemas jurídicos contemporáneos son complejos, diversos y no existe un orden idílico, el caso de las PcD no existen esfuerzos significativos para integrar los diferentes objetivos legales a nuestro sistema jurídicos.

Finalmente, es posible verificar una tendencia de resistencia en la aplicación de derecho internacional por parte de los jueces de primera instancia en lo referido a los casos de discriminación de la Ley 20.609. En efecto, la estructura propia de los tribunales civiles, sus objetivos y campo de aplicación hacen que sea un terreno hostil para la integración de derecho internacional en sus sentencias. Así, se comprueba como cambia el comportamiento de los tribunales civiles cuando deciden integrar tratados internacionales de derechos humanos en sus fallos en lo que concierne a los casos discapacidad ${ }^{25}$.

En conclusión, y a partir de lo comentado en este trabajo, se puede colegir que la noción de accesibilidad es un elemento clave en el análisis de la discriminación en las PcD. Con todo, si bien se observa una incorporación de los principios jurídicos de la Convención de las Personas con

24 ÍDEM.

$25 \mathrm{Al}$ respecto, en las sentencias del $5^{\circ}$ Juzgado CIVIL de Valparaíso rol № $1912-2016$ y $17^{\circ}$ JuzGado CiVIL de Santiago, rol No 27742-2016. 
Discapacidad en la actividad adjudicativa de la Corte Suprema, se evidencia una series dificultades para las PcD en litigación de sus derecho. Tal hecho debe ser mirado con preocupación por la comunidad jurídica, pues, el litigio ante la Corte Suprema devela un síntoma de una defectuosa e imperfecta incorporación e implementación de los principios y directriz de la Convención de las Personas con Discapacidad en nuestras diversas instituciones. Tales circunstancias nos conduce a problemas de acceso a la justicia en las $\mathrm{PcD}$, dado que no todas las discapacidades son simétricas, pues algunas discapacidades permiten mayores niveles de organización e impacto en el sistema político. Lo anterior, es fundamental al momento de embarcarse en un extenso litigio.

\section{Bibliografía}

Alexy, Robert (1993). Teoría de los Derechos Fundamentales. Madrid: Centro de Estudios Constitucionales.

BRODERICK, Andrea (2019). "Of rights and obligation: the birth of accessibility", The International Journal of Human Rights, pp. 1-21.

DE AsIs, Rafael (2013). Sobre discapacidad y derechos. Madrid: Dykison.

- (2016). "Reasobleness in the concept of reasonable accommodation", The The Age of Rights Journals, No 6. (2016): 42-59.

DíAZ, Iván (2013): "Ley chilena contra la discriminación. Una evaluación desde los derechos internacional y constitucional”, en Revista Chilena de Derecho (vol. 40, $\mathrm{N}^{\circ}$ 2), pp. 635-668

DíAZ dE VALdÉs, José Manuel, (2013): “¿Es la Ley Zamudio verdaderamente una ley general antidiscriminación?", en Actualidad Jurídica (año 14, $\mathrm{N}^{\circ} 28$ ), pp. 279-297.

-. (2108). "Análisis cuantitativo de la acción especial antidiscriminación de la Ley Zamudio". En: Actualidad Jurídica.

-. (2019). Igualdad y No Discriminación. Santiago: Editorial Tirant Lo Blanch.

DoYLE, Oran (2007). "Direct Discrimination, Indirect Discrimination and Autonomy", en Oxford Journal of Legal Studies, Vol. 27, No. 3, pp. 537-553. 
CAYO, Luis (2012). "La configuración jurídica de los ajustes razonable", en Revista 2003-2012: 10 años de Legislación sobre no Discriminacion en Personas con Discapacidad en España. Estudios en homenaje a Miguel Ángel Cabra de Luna. Comité Español de Representantes de Personas con Discapacidad (Cermi) (N55).

Comité de las Personas con Discapacidad (2013)." Observación general sobre el artículo 9: accesibilidad". [Fecha de consulta: 26 de febrero de 2020]. [Disponible en: https://undocs.org/pdf?symbol=CRPD/C/11/3].

- (2016). "Observación general núm. 3, sobre las mujeres y las niñas con discapacidad". [Fecha de consulta: 26 de febrero de 2020]. [Disponible en: http://docstore.ohchr.org/SelfServices/FilesHandler. ashx? enc $=6$ QkG1d\%2FPPRiCAqhKb7yhsnbHatvuFkZ\%2Bt93Y3D\%2Baa2oLCHc5Ye5y0yX37Gpo\%2FkmBZI1QeKTg7cNEuS\%2FzKc3xGM7PD4P8YrjsNLHbSyyH3\%2BpDNGpobvX\%2B6Zw74L1Z2GWT]

Esparza-Reyes, Estefanía; Díaz, Francisco (2019). “Los mecanismos jurídicos de lucha contra la discriminación: aportaciones para la configuración del derecho antidiscriminatorio", en Revista de Derecho Político (mayo-agosto 2019, N¹05), pp.57-79.

FINSTERBusch, Christian (2007). "La extensión de los ajustes razonables en el derecho de las personas en situación de discapacidad de acuerdo al enfoque social de derechos humanos". Revista lus Et Praxis, 22(2), $227-252$.

GrzedA, Jerzy (1994). "Disability and Discrimination a UK Perspective", Business Ethics 3 (3):145-147.

MuÑOz, Fernando (2015): "Estándares conceptuales, cargas procesales y reparación en el litigio antidiscriminación. Análisis crítico de la jurisprudencia sobre Ley Zamudio entre 2012 y 2015", en Revista de Derecho de la Universidad de Valdivia (vol. 27, № 2), pp. 145-167.

OlIVER, Michael (1996). Understanding Disability: From Theory to Practice. New York: Macmillan Education.

Palacios, Agustina (2004). "El derecho a la igualdad de las personas con discapacidad y la obligación de realizar ajustes razonables”. En: Campoy, Ignacio. Los derechos de las personas con discapacidad : pers- 
pectivas sociales, políticas, jurídicas y filosóficas. Madrid: Dykinson. Rosales, Cecilia (2014): "Acción de no discriminación arbitraria”, en AA. VV., en Acciones protectoras de derechos fundamentales (Santiago, Thomson Reuters), pp. 255-296.

VIAL, Tomás (2013): "La Nueva Ley Antidiscriminación: Propuestas para avanzar en su Perfeccionamiento", en AA.VV., Anuario de Derechos Humanos $\mathrm{N}^{\circ} 9$ (Santiago, Universidad de Chile), pp. 183-191.

\section{Anexo}

\begin{tabular}{|c|c|c|}
\hline 1. & Fecha: & 25 de julio de 2018 \\
\hline 2. & Recurrente: & Jeannette Lucía Sandoval Camarada \\
\hline 3. & Recurrido: & Empresa de Transportes de Pasajeros Metro S.A. \\
\hline 4. & Recurso: & Recurso de Casación en el Fondo \\
\hline 5. & Sala: & Cuarta sala \\
\hline 6. & Redacción: & Gloria Ana Chevesich Ruiz \\
\hline 7. & Rol: & $41.388-17$ \\
\hline 8. & Integración: & $\begin{array}{l}\text { Ministros (a) Sr. Ricardo Blanco H., Sra. Gloria Ana Chevesich R. y } \\
\text { Sra. Andrea Muñoz S. y los Abogados Integrantes Sr. Álvaro Quintani- } \\
\text { Ila P. y Sra. Leonor Etcheverry C. }\end{array}$ \\
\hline 9. & Votación: & Unánime \\
\hline 10. & Resuelve: & Acoge \\
\hline 11. & $\begin{array}{l}\text { Grado de } \\
\text { Discapacidad: }\end{array}$ & Física de un $70 \%$ \\
\hline
\end{tabular}

\section{Resumen}

En circunstancias que la recurrente y demandante del caso, quien posee una discapacidad física de un $70 \%$, se encontraba realizando un viaje diario en el Metro de Santiago, este se detiene bruscamente debido a un aviso de un posible artefacto explosivo, a lo cual el operador de la estación comunica que se iniciará un operativo de seguridad destinado a evacuar a los pasajeros. Producto de la anterior, personal de Metro de Santiago procedió a evacuar a los pasajeros, entre los cuales se encontraba la recurrente y demandante del caso, pero esta última no pudo hacerlo debido a que la salvaescala, artefacto de accesibilidad para las 
Personas con Discapacidad instalado en el metro, se encontraba en mal estado, lo cual naturalmente provocó que no pudiera evacuar junto a los demás pasajeros, permaneciendo dentro de la estación de metro durante el operativo.

\section{Sentencia}

Considerando $4^{\circ}$ : Que, en ese contexto, no es atendible la única alegación que la demandada formuló al informar al tenor de la demanda, en el sentido que no estaba obligada a dar cumplimiento a la normativa nacional e internacional a que se ha hecho referencia, porque el artículo primero transitorio de la Ley $\mathrm{N}^{\circ} 20.422$, publicada en el Diario Oficial el 10 de febrero de 2010, le otorgó un plazo de ocho años a contar de dicha data para hacer accesible y utilizable en forma autovalente y sin dificultad por personas con discapacidad, especialmente por aquellas con movilidad reducida, el acceso a los medios de transporte público de pasajeros; más aún si, como se señaló, el artículo 82 de dicha ley no derogó el artículo 21 de la Ley $\mathrm{N}^{\circ}$ 19.284. Con todo, aceptar dicho planteamiento implica que las personas discapacitadas estuvieron prácticamente privadas de usar la red subterránea de transporte público de la demandada hasta el mes de febrero de 2018 , lo que se traduce en una grave conculcación a los derechos y libertades fundamentales de que son titulares y a la normativa internacional mencionada que precisamente persigue promoverlos, protegerlos y asegurarlos.

Considerando $5^{\circ}$ : Que la demandada al quedar en evidencia que la estación de trenes a que se ha hecho referencia tiene instalado un salva escala, señaló, como consta a fojas 58 , que solo permite recorrer el tramo de escala existente entre el andén y las boleterías, no así hacia la superficie, lo que será posible cuando se construya un ascensor, por lo que se encuentra desactivado, fuera de uso, y que no tiene ningún sentido activarlo. Agregó que su instalación se efectuó anticipadamente a su uso efectivo, solo porque por razones de economía adquirió, importó e instaló, en una misma oportunidad y en todas las estaciones, dichos adminículos que son mecanismos parciales de acceso. Dicha aseveración 
no solo importa dejar de manifiesto que incumplió la obligación que le impuso el artículo 21 de la Ley $N^{\circ} 19.284$, dado que no solo no instaló el sistema apropiado que permitiera acceder desde la superficie al andén y viceversa, sino que el que emplazó y con el que se lograba un acceso parcial lo mantuvo inutilizado, lo que se traduce, en los términos del artículo 2 de la Convención de las Naciones Unidas sobre los Derechos de las Personas con Discapacidad, en una forma de discriminación, específicamente, la denegación de ajustes razonables . Corrobora dicha conclusión la circunstancia que ninguna probanza rindió para acreditar lo que señala, esto es, la concurrencia de razones económicas que lo autorizaron para obrar como lo hizo. Al respecto, se debe tener presente que la misma disposición utiliza la expresión "ajustes razonables”, concibiéndola como "...las modificaciones y adaptaciones necesarias y adecuadas que no impongan una carga desproporcionada o indebida, cuando se requieran en un caso particular, para garantizar a las personas con discapacidad el goce o ejercicio, en igualdad de condiciones con las demás, de todos los derechos humanos y libertades fundamentales".

En consecuencia, la conducta de la demandada debe ser calificada de discriminatoria por motivos de discapacidad, entendiendo por tal, conforme los términos del artículo 2 de la citada convención, “...cualquier distinción, exclusión o restricción por motivos de discapacidad que tenga el propósito o el efecto de obstaculizar o dejar sin efecto el reconocimiento, goce o ejercicio, en igualdad de condiciones, de todos los derechos humanos y libertades fundamentales en los ámbitos político, económico, social, cultural, civil o de otro tipo. Incluye todas las formas de discriminación, entre ellas, la denegación de ajustes razonables".

\section{Decisión de la Corte}

Considerando $8^{\circ}$ : Que, entonces, si la demandante se desplaza en silla de ruedas por tener una discapacidad física equivalente al $70 \%$, y por el episodio acaecido el 19 de mayo de 2016, alrededor de las 8:00 A.M., se detuvo el servicio de transporte en la Estación Santa Isabel del tren subterráneo del Metro de Santiago y se evacuó a todos los pasajeros 
por el potencial riesgo existente para los mismos, mas no a ella, precisamente porque la demandada incumplió la normativa legal $y$, en todo caso, mantenía inutilizado el salva escala que habría permitido que accediera al recinto de la boletería, sin que haya alegado, menos probado, la concurrencia de una justificación razonable en los términos expuestos en el inciso final del artículo 2 de la Ley $\mathrm{N}^{\circ} 20.609$, se configuró un acto de discriminación, en la modalidad de distinción que, en los términos del mensaje de la Ley $N^{\circ} 20.609$, es una diferenciación en base a alguna particularidad, la discapacidad, que causó perturbación en el ejercicio legítimo de los derechos fundamentales ya señalados y de que es titular la demandante.

Por estas consideraciones y de conformidad, además, con lo dispuesto en el artículo 12 de la Ley $N^{\circ} 20.609$ y en los artículos 186 y siguientes del Código de Procedimiento Civil, se revoca la sentencia apelada de trece de marzo de dos mil diecisiete, escrita a fojas 61 y siguientes, y acogiéndose la demanda se declara que la demandada, Empresa de Transportes de Pasajeros Metro S.A., representada por don Rodrigo Azócar Hidalgo, incurrió en un acto de discriminación arbitraria respecto de doña Jeannette Lucía Sandoval Camarada, en razón de su discapacidad, por lo que deberá adoptar todas las medidas necesarias para no incurrir nuevamente en el mismo. Además, se la condena al pago de una multa equivalente a treinta unidades tributarias mensuales, a beneficio fiscal, que deberá pagar dentro del plazo de cinco días, a contar de la data en que la presente sentencia quede ejecutoriada, con costas. 
